\title{
Power Generation Expansion Planning Model Towards Low-Carbon Economy and Its Application in China
}

\author{
Qixin Chen, Chongqing Kang, Senior Member, IEEE, Qing Xia, Senior Member, IEEE, and \\ Jin Zhong, Member, IEEE
}

\begin{abstract}
Climate change poses a huge threat to human welfare. Hence, developing a low-carbon economy has become a prevailing and inevitable trend. Decarbonization of power generation, especially converting the current power mix into a low-carbon structure, will be a critical option for $\mathrm{CO}_{2}$ emission mitigation. In this paper, an integrated power generation expansion (PGE) planning model towards low-carbon economy is proposed, which properly integrates and formulates the impacts of various low-carbon factors on PGE models. In order to adapt to the characteristics of PGE models based on low-carbon scenario, a compromised modeling approach is presented, which reasonably decreases complexities of the model, while properly keeping the significant elements and maintaining moderate precision degree. In order to illustrate the proposed model and approach, a numerical case is studied based on the background of China's power sector, making decisions on the optimal PGE plans and revealing the prospects and potentials for $\mathrm{CO}_{2}$ emission reduction.
\end{abstract}

Index Terms-Carbon capture and storage, $\mathrm{CO}_{2}$ emission, global climate change, low-carbon economy, modeling approach, power generation expansion planning model.

\section{INTRODUCTION}

C LIMATE change resulted from greenhouse gas (GHG) poses a huge threat to human welfare [1]-[3], and $\mathrm{CO}_{2}$ contributes $77 \%$ of GHG effects. On this basis, since 2003 when the UK Energy White Paper [4] was published, developing a low-carbon economy has gradually become prevailing and inevitable. Being a major $\mathrm{CO}_{2}$ emission source, decarbonisation in power sector is critical and fundamental in confronting the challenges of global warming. However, with long in-service lives as several decades, investments on conventional fired-based generators will lead to intensive "carbon lock-in" effect in the future [5]. Hence, it is critical to convert the current power mix into a low-carbon structure.

Manuscript received May 30, 2009; revised October 21, 2009. First published January 08, 2010; current version published April 21, 2010. This work was supported in part by Grant of State Key Lab of Power Systems (SKLD09M09), Scientific and Technical Project of State Grid, The Project Sponsored by Scientific Foundation for Returned Overseas Chinese Scholars, Ministry of Education ([2009]1001), and Program for New Century Excellent Talents in University (NCET-07-0484). Paper no. TPWRS-00398-2009.

Q. Chen, C. Kang, and Q. Xia are with the State Key Lab of Power Systems, Department of Electrical Engineering, Tsinghua University, Beijing 100084, China (e-mail: cqx@mails.tsinghua.edu.cn).

J. Zhong is with the Department of Electrical and Electronic Engineering, University of Hong Kong.

Color versions of one or more of the figures in this paper are available online at http://ieeexplore.ieee.org.

Digital Object Identifier 10.1109/TPWRS.2009.2036925
One of the elements in power generation expansion (PGE) is to determine the optimal mix of power generation. Generally, PGE is a large-scale mixed integer nonlinear programming problem [6], making decisions on which, where, and when new power plants should be built over a given time horizon [7]. However, with the emerging of more evidences on climate changing and $\mathrm{CO}_{2}$ emission reduction, impacts on PGE planning incurred by various low-carbon factors should be properly considered. In [6], [8] and [9], emission control is regarded as an additional constraint in PGE model; the impacts of large-scale utilization of wind power on power system planning are evaluated in [10]; market penetrations of clean coal and carbon capture and storage technologies (CCS) are assessed considering various political as well as economical instruments in [11] and [12], respectively. However, these researches just focus mostly on some special kinds of low-carbon factors; it is necessary to develop a comprehensive PGE planning model, which appropriately integrates all kinds of low-carbon factors, including carbon trading mechanisms, low carbon generation technologies, reduction obligations, carbon tax, etc. For this purpose, a compromised modeling approach is proposed, which reasonably decreases complexities of the model, while properly keeping the significant elements and maintaining moderate precision degree. The model is denoted as low carbon power generation expansion (LCPGE) for short.

China is one of the major $\mathrm{CO}_{2}$ emitting countries due to its coal-dominated (more than $75 \%$ ) power mix. As estimation, the growth rate in GDP as well as power consumptions will remain at high level continuously in the near future. Therefore, it is of great significance to assess the potential of $\mathrm{CO}_{2}$ emission reduction and reveal the optimal low carbon roadmap in China's power sector. For this purpose, a numerical case based on China's power sector is studied. The case study could provide better illuminations on the proposed LCPGE model and the modeling approach as well.

Generally, the paper is organized as below: The framework of LCPGE model is described in Section II; the modeling approach is introduced in Section III; formulations of the model are presented in Section IV; an illustrative example based on China's power sector applying LCPGE model is analyzed in Section V; and Section VI provides the conclusion.

\section{FRAMEWORK OF LCPGE MODEL}

Low-carbon factors would impose great impacts on PGE models. Firstly, carbon prices or taxes would be imposed 


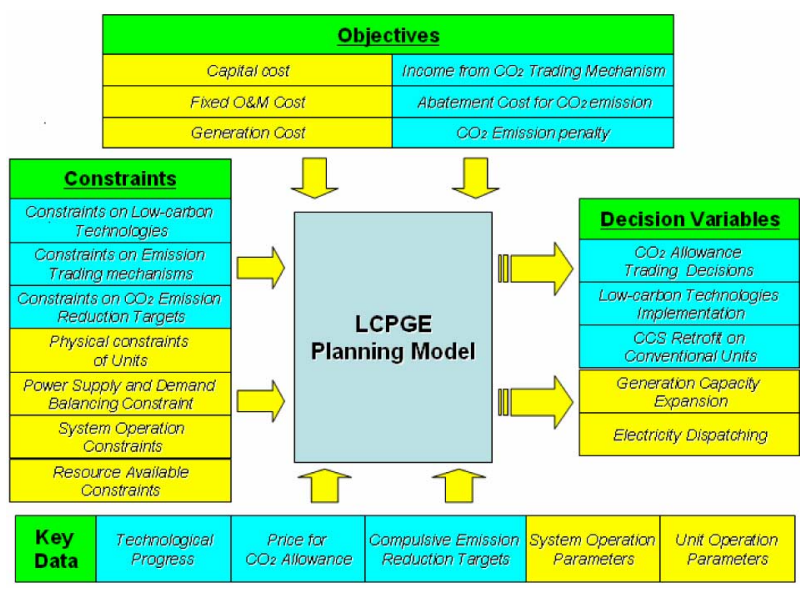

Fig. 1. Framework of the LCPGE model.

on $\mathrm{CO}_{2}$ emission, leading to changes in production cost of electricity. Thus, the merit order of power plants in generation dispatching would be adjusted due to the differences of $\mathrm{CO}_{2}$ intensities among plants. Secondly, $\mathrm{CO}_{2}$ allowances might be allocated to plants freely or by auction, and the plants have to surrender allowances for their emitted $\mathrm{CO}_{2}$; then trading mechanisms would be established for allowance transactions. These elements would affect the decisions on PGE planning. Thirdly, various low-carbon technologies would be introduced as major options to mitigate carbon emissions. Moreover, in order to restrict excessive emission, reduction obligations set in international stage and domestic compulsive reduction targets may be imposed on power sector.

The framework of LCPGE model is given in Fig. 1; shallow modules represent regular components of PGE models; and the dark ones denote the newly introduced components.

As shown in Fig. 1, the objective of LCPGE is to minimize the total cost, including not only capital investments, fixed O\&M costs and generation costs, but also costs related to $\mathrm{CO}_{2}$ emission, such as abatement costs, allowance trading incoming and emission penalty if emitting excessive $\mathrm{CO}_{2}$. Besides capacity expansion plans and generation dispatching plans, the outputs of LCPGE model include additional decision variables on low-carbon technology implementation, CCS retrofit plans of conventional fired-based plants, and $\mathrm{CO}_{2}$ allowance trading decisions in carbon markets. Furthermore, apart from the regular ones, constraints related to low-carbon technologies, $\mathrm{CO}_{2}$ trading mechanisms and reduction targets are extra introduced as well.

\section{MODELING APPROACHES}

\section{A. Discussions on the Existing PGE Modeling Approaches}

There are mainly two categories of modeling approaches in PGE depending on the precise degree of modeling, which are named "micro-approach" and "macro-approach".

"Micro-approach" is utilized in [6]-[8] and [10], by which PGE models are set in mixed-integer style. " $0-1$ " decision variables are introduced to denote the expansion options of candidate plants, which are differentiated by their types, capacities and locations. Power production simulations are adopted for generation dispatching, and evaluations on operation reliability might even be included. Meanwhile, complex constraints related to the operation of transmission network and generators are imposed. With "micro-approach", the result of PGE planning could be quite definite and fine, and the optimality of the model is always perfect if all details and data are set at high level of exactness. However, this approach is not appropriate for LCPGE as there are great uncertainties in low-carbon scenario. For example, the expectation of $\mathrm{CO}_{2}$ emission reduction is still being discussed; the prospects of low-carbon technologies are not quite clear; and the progress of $\mathrm{CO}_{2}$ trading mechanisms as well as carbon prices are hard to be foreseen. Therefore, it is not realistic and necessary to adopt "micro-approach" in LCPGE models, as complexities will be increased to a great extent while the improvements in precision are not equivalent.

"Macro-approach" is utilized in [9], [11] and [12]. Compared with "micro-approach", "macro-approach" is usually based on linear equilibrium, with lots of simplifications to reduce complexities of modeling. Plenty of available models based on "macro-approach" have been developed, such as MARKAL and META Net, applying "bottom-up" modeling methods. However, these models are always not established for PGE specially; most of them are applied to analyze the planning of the whole economy/energy system based on multi-sector scenarios; power plants are taken as identical elements of the models with standard definitions of inputs and outputs, like oil refinery plants in petroleum sector and vehicles in transportation sector. Key characteristics of involved activities in power sector are ignored, and the relations between PGE and power system operation are not properly considered. Therefore, PGE planning results based on "macro-approach" are always too macroscopic and roughly.

\section{B. New Approach Developed for the Modeling of LCPGE}

In order to adapt to the characteristics of LCPGE model, a new modeling approach is developed.

Firstly, capacity expansion is no longer considered unitbased. A set of power plants with the same type and location would be represented by an integrated decision variable. This scheme is referred as "plant-set-based". Thus, the integral " $0-1$ " decision variables could be excluded. This treatment would reduce the precision of modeling to a lesser extent. Though installed capacities are of specific discrete values, they are also diversified, for example, $0.9,1.5$ and $3 \mathrm{MW}$ for wind turbines. The combination of these discrete values could be taken as a continuous number approximately, especially when the total planned capacities are much more than that of any single unit.

Secondly, power production simulations are not included in LCPGE model; instead, a scheme of generation dispatching among different sets of plants is introduced. New decision variables are integrated, denoting the dispatched generation outputs to different sets of plants at a given time-scale. Generation dispatching is one of the most critical processes in determining the total generation cost and the competitiveness among different sets of plants. By adopting the scheme, plants with low production costs would be scheduled on in priority. Therefore, cost-profit features of the model could be mostly reflected. 
Meanwhile, modeling works could be largely cut by avoiding the nonlinear relations within production simulations. The scheme is effective for planning cases with long time horizons and great uncertainties. Plenty of additional operation data are always required for production simulations. In scenarios with great uncertainties, it is difficult to ensure the accessibility and exactness of data. The improvements in modeling might probably be offset by the in-exactness in data obtaining. However, in the proposed scheme, the scale of required data is largely cut, while the major relations between power generation and system operation are retained. Hence, the scheme achieves a proper equilibrium between the precision of model settings and the exactness of basic data.

Moreover, as production simulation is simplified and decision variables are set "plant-set-based", it is unnecessary to consider physical connections among generators in transmission network. Instead, caps on export/import capacity between regions are introduced. In this case, constraints based on Kirchhoff Laws in power delivery could be disregarded.

With the new modeling approach, general mixed integer nonlinear PGE planning problems could be formulated as standard linear models. In this case, the augment in model scale would not largely aggravate the difficulties in finding optimal solutions. Therefore, the proposed LCPGE model is of good adaptability and could be easily expanded to deal with various new factors and more complex scenarios. It should be noted that uncertainties in low carbon factors would not be formulated by integrating probability distributions in order to better concentrate on the paper's main topic. Instead, plenty of multi-scenario analysis would be carried out in case study.

\section{Formulations OF LCPGE Model}

\section{A. Decision Variables}

Four groups of decision variables are presented in the model:

$X_{m, y}^{N} \quad$ expanded capacity of plant set $m$ in year $y$;

$X_{m, y}^{R} \quad$ CCS Retrofit capacity of plant set $m$ in year $y$;

$X_{m, y}^{G} \quad$ yearly generation dispatched to plant set $m$ in year $y$;

$X_{n, y}^{E} \quad$ overall traded allowance in mechanism $n$ in year $y$.

Besides, carbon prices are taken as exogenous variables by assuming perfect competition in trading mechanisms.

\section{B. Objective Function}

The scheme minimizes the aggregate of the overall cost:

$$
\begin{aligned}
\min C=\sum_{y \in Y}(1+r)^{-y}\left(C_{y}^{I}+C_{y}^{R}+C_{y}^{O}\right. & +C_{y}^{G} \\
& \left.-C_{y}^{E}+C_{y}^{P}\right)
\end{aligned}
$$

where $C_{y}^{I}, C_{y}^{R}, C_{y}^{O}, C_{y}^{G}, C_{y}^{E}$ and $C_{y}^{P}$. respectively. denote capital investments, CCS retrofit expenses, O\&M costs, generation costs, $\mathrm{CO}_{2}$ trading income and emission penalty in year $y$ for the system:

$$
\left\{\begin{array}{l}
C_{y}^{I}=\sum_{m \in U} X_{m, y}^{N} \cdot c_{m, y}^{I} \\
C_{y}^{R}=\sum_{m \in U}^{m} X_{m, y}^{R} \cdot c_{m, y}^{R} \\
C_{y}^{O}=\sum_{m \in U} U_{m, y} \cdot c_{m, y}^{O} \\
C_{y}^{G}=\sum_{m \in U}^{m} X_{m, y}^{G} \cdot c_{m, y}^{G} \\
C_{y}^{E}=\sum_{n \in T}^{m} X_{n, y}^{E} \cdot\left(\pi_{n, y}^{E}-c_{n, y}^{T}\right) \\
C_{y}^{P}=E_{y}^{P} \cdot \pi_{y}^{E}
\end{array}\right.
$$

where $c_{m, y}^{I}, c_{m, y}^{R}, c_{m, y}^{O}$ and $c_{m, y}^{G}$ denote corresponding unit cost (per $\mathrm{kW} / \mathrm{kWh}$ ) according to their superscript. $Y$ is set of all planned years; $r$ denotes deflating rate; $\pi_{n, y}^{E}$ indicates $\mathrm{CO}_{2}$ allowance price in mechanism $n ; c_{n, y}^{T}$ denotes the related transaction cost consisting of various commissions; $E_{y}^{P}$ represents exceeded emission allowance while $\pi_{y}^{E}$ is the penalty; $U_{m, y}$ denotes the total in-service capacity of plant set $m$; $U$ is set of all involved plants; and $T$ is set of all trading mechanisms.

"Learning effect" leads to declining trend on various costs. It is treated as exogenous, constant reduction rate over time in this paper [13], [14]:

$$
c_{m, y}^{I}=c_{m, 0}^{I} \cdot\left(1-r_{m}^{I}\right)^{y-y_{0}}
$$

where $y_{0}$ is the starting year of planning; $c_{m, 0}^{I}$ is the base investment in $y_{0}$; and $r_{m}^{I}$ is the cost reduction rate in technology $m$. The terms in (3) could be replaced by terms related to O\&M costs or production costs as well. $U_{m, y}$ is expressed as (4) at the bottom of the page.

In (4), $U_{m, 0}$ is the in-service capacity of plant set $m$ in $y_{0}$ and $U_{m, i}^{D}$ denotes the retired capacity. $U_{N}$ is set of plants that have not been retrofitted; while $U_{R}$ is set of plants retrofitted.

$$
U_{m, y}= \begin{cases}U_{m, 0}+\sum_{i=1}^{y} X_{m, i}^{N}-\sum_{i=1}^{y} X_{m, i}^{R}-\sum_{i=1}^{y} U_{m, i}^{D} & m \in U_{N} \\ U_{m, 0}+\sum_{i=1}^{y} X_{m, i}^{R}-\sum_{i=1}^{y} U_{m, i}^{D} & m \in U_{R}\end{cases}
$$


Meanwhile, $E_{y}^{P}$ is formulated as follows:

$$
\begin{aligned}
E_{y}^{P}=\max \left(0, \sum_{m \in U}\left(X_{m, y}^{G} \cdot e_{m, y}^{G}\right)\right. & \\
& \left.-\sum_{n \in T} \sum_{i=1}^{\max \left(Y_{n}^{P}, y-1\right)} X_{n, y-i}^{E}-E_{y}^{T}\right)
\end{aligned}
$$

where function $\max$ indicates getting the bigger value within the elements in the bracket. $e_{m, y}^{G}$ denotes emission intensity of plant set $m ; Y_{n}^{P}$ is the length of validity period for allowance contracts; and $E_{y}^{T}$ is cap on allowances allocated to the system, which might be decided by the government.

\section{Constraints}

1) Bounds on Yearly Power Generations of Plants: Year is used as a critical timescale in PGE models with long time horizons. A conception: "yearly generating hour" (YGH) is introduced to equivalently represent the utilization of power generators in average sense, which equals to annual cumulative generation outputs divided by the rated capacity of generators. Upper and lower limits should be imposed on the generation dispatching scheme:

$$
H_{m, y}^{L} \cdot U_{m, y} \leq X_{m, y}^{G} \leq H_{m, y}^{U} \cdot U_{m, y}
$$

where $H_{m, y}^{U}$ and $H_{m, y}^{L}$ is the upper and lower bounds of YGH. $H_{m, y}^{U}$ expressed the same meaning as capacity factors. it is always set lower than the theoretical maximum value, $8760 \mathrm{~h}$, for physical issues and political issues. Though could be as low as zero when plants are not dispatched completely, $H_{m, y}^{L}$ is always set as special nonzero values for two reasons. Firstly, to accord with protection policies on some kinds of plants; secondly, to keep plants in-service or ready for reserve.

The introduction of CCS technology would decrease net efficiency of power plants and lead to "capacity penalty". The penalty is treated by means of discounting equivalent YGH:

$$
H_{m 1, y}^{U}=H_{m 2, y}^{U} /\left(1+\Delta f_{y}^{L}\right)
$$

where $H_{m 1, y}^{U}$ and $H_{m 2, y}^{U}$ denote the upper limits of YGH for plants with and without CCS; and $\Delta f_{y}^{L}$ denotes the loss in efficiency after implementing CCS.

2) Requirements on Available Reserve Capacities: Maintaining adequate available reserve capacities could help to mitigate the risk of unplanned outage and fluctuations in power outputs. Therefore, at the timescale of year, proper headroom of average YGH should be kept:

$$
H_{y}^{A} \cdot \sum_{m \in U} U_{m, y} \geq D_{y}
$$

where $D_{y}$ is power demand in year $y ; H_{y}^{A}$ is the maximum average YGH for all plants involved; $H_{y}^{A}$ is related to power mix, and could be fixed according to historical statistics.
3) Caps on Export and Import Capacities Between Regions:

$$
D_{r, y}-\operatorname{Im}_{r, y}+\mathrm{Ex}_{r, y} \leq \sum_{m \in R e g} X_{m, y}^{G}
$$

where $D_{r, y}$ represents power demand of region $r$ in year $y$; $\mathrm{Ex}_{r, y}$ and $\mathrm{Im}_{r, y}$ denotes the caps of exporting and importing capacities, which are fixed according to the total available transmission capacities between regions. Reg is set of all involved regions.

4) Equation on Power Demand and Supply: Electricity that generated by plants should be equal to the anticipated power demand annually, which is

$$
\sum_{m \in U} X_{m, y}^{G}=D_{y}
$$

5) Limits on Cumulative Capacities: Cumulative capacities for every set of plants should be nonnegative, indicating that annual retrofitted capacities should not surpass the sum of existing and newly expanded capacities:

$$
U_{m, y} \geq 0
$$

The expression of $U_{m, y}$ is given in (4).

6) Maximum Domestic Fuel Supply and Import Proportion: Fuel consumption should not exceed the maximum domestic production to a certain proportion for the purpose of ensuring energy supply security and reducing price risks:

$$
\sum_{m \in F_{n}}\left(X_{m, y}^{G} \cdot f_{m, y}\right) \leq\left(1+r_{n, y}\right) \cdot F_{n, y}^{L}
$$

where $f_{m, y}$ is fuel consumption intensity of plant set $m ; F_{n, y}^{L}$ is the maximum available domestic production of fuel $n ; r_{n, y}$ is the maximum import proportion; and $F_{n}$ is set of all plants based on fuel $n$.

7) Caps on Exploitable Capacity: Hydro, wind and other renewable generations are not fuel-based. However, caps should also be imposed on their annual exploitable capacities, which may come from the maximum scale of available natural resources, inadequacy of technology development, or maximum production ability of equipments:

$$
U_{j, 0}+\sum_{i=1}^{y} \sum_{m \in R_{j}} X_{m, i}^{N} \leq \sum_{i=1}^{y} R_{j, i}^{L}
$$

where $R_{j, i}^{L}$ is caps of exploitable capacity for resource $j$ in year $i ; R_{j}$ is set of plants based on resource $j$; and $U_{j, 0}$ is the current in-service capacity of plants based on resource $j$.

8) First-Introduction Date of Technology: New technology would be available for commercial utilization after a specific introduction date, so we have

$$
X_{m, y}^{N}=0 \quad \forall y \leq Y_{m}^{A}
$$

where $Y_{m}^{A}$ is the first introduction date of technology $m$.

9) Caps on Expansion Capacity in Transition Period: Transition period of new technologies indicates a specific length of 
time after their first introduction dates, which always takes several years. In the transition period, caps are imposed on the expansion capacities, allowing for necessary time before the technologies could be utilized at large-scale:

$$
X_{m, y}^{N} \leq \operatorname{Cap}_{m, y}^{\max } \quad \forall Y_{m}^{A} \leq y \leq Y_{m}^{A}+Y_{m}^{T} .
$$

$\mathrm{Cap}_{m, y}^{\max }$ is the cap and $Y_{m}^{T}$ is the length of the period.

10) Constraints on $\mathrm{CO}_{2}$ Emission: Three kinds of constraints could be imposed on total $\mathrm{CO}_{2}$ emission, emission intensity, or reduction rate, respectively. Considering allowance trading, the constraints are expressed as (16) at the bottom of the page, where $E^{T}$ is the upper bound of total emission for the whole planning period; $e_{y}^{T}$ is the targeted emission intensive, $E_{0}^{T}$ is the emission level in benchmark year, $r_{y}^{E}$ is the reduction rate; and $Y_{E}$ is set of years on which $r_{y}^{E}$ is imposed.

11) Caps on Overall Tradable Allowance in $\mathrm{CO}_{2}$ Trading Mechanisms: Caps should be imposed on tradable $\mathrm{CO}_{2}$ allowance when they should be real delivered in trading mechanisms, for example, in clean development mechanism (CDM). It means that the maximum traded volume could not surpass the in-hand available allowances. The constraints are imposed on the planned system, but not any single power plant or corporation:

$$
X_{m, y}^{E} \leq \sum_{m \in T_{C}} p_{m, y}^{\max } \cdot X_{m, y}^{G} \cdot\left(e_{m, y}^{G}-e_{m, y}^{B}\right)
$$

where $e_{m, y}^{B}$ is the benchmark of emission intensity; $T_{C}$ is set of plants qualified in mechanisms. $p_{m, y}^{\max }$ denotes the maximum tradable proportion within all qualified allowances, which is decided by size and fluidity of carbon market.

12) Constraints on Fluctuations of Yearly Expanded Capacity: The process of capacity expansion should not fluctuate violently over years in order to keep a relatively steady money flow of the planners, especially when the models are built at country-wide level. $r^{I}$ is set as fluctuation rate of expanded capacity between years. The constraint could be expressed as

$$
\begin{aligned}
& \left(1-r^{I}\right) \cdot \sum_{m \in U} X_{m, y-1}^{N} \\
& \quad \leq \sum_{m \in U} X_{m, y}^{N} \leq\left(1+r^{I}\right) \cdot \sum_{m \in U} X_{m, y-1}^{N} .
\end{aligned}
$$

13) Nonnegativity of Some Decision Variables:

$$
X_{m, y}^{N}, X_{m, y}^{R}, X_{m, y}^{G} \geq 0 .
$$

TABLE I

Capacity EXPansion Plans of Non-Decisive Types of Plants

\begin{tabular}{cccc}
\hline Plant type (in GW) & $\mathbf{2 0 1 0}$ & $\mathbf{2 0 2 0}$ & $\mathbf{2 0 3 0}$ \\
\hline Conventional coal-fired & 420 & 364 & 280 \\
Gas-fired & 23 & 50 & 80 \\
Oil-fired & 10 & 12 & 15 \\
Hydro & 194 & 328 & 430 \\
Nuclear & 10.5 & 70 & 140 \\
Other renewables & 2 & 10 & 30 \\
\hline
\end{tabular}

In the case study, the basic settings above would be referred as "normal scenario".

\section{Case Study on China's Power Sector}

Being a standard linear programming problem, the LCPGE model can be solved by using some software packages, such as MATLAB, Lingo, Xpress, etc. The case model is solved by MATLAB on a Notebook with Pentium-M $(2.0 \mathrm{GHz})$ processor and $1 \mathrm{G}$ MB of DDR-RAM.

The model is implemented to gain insights into the optimal PGE plan for China's power sector and reveal the prospects of related $\mathrm{CO}_{2}$ emission covering a time frame from 2011 to 2030. PGE up to 2010 is not decided as most of the candidate plants for that period have already been committed. The case is established at country-wide level, regardless of multi-regions.

\section{A. Low-Carbon Factors}

1) Candidate Power Plants: Three options of coal technologies are applied: super-critical (SC), ultra-supercritical (UC), and IGCC. CCS is included as well; however, there should be a considerable period of time before CCS could be commercialized at large scale. In order to avoid "carbon lock-in" effect, being carbon capture ready (CCR) becomes an important option for normal fired-based plants. Carbon capture plants could be built directly; or be CCR first, then retrofit. Moreover, wind is included as a typical option of renewables, ignoring others.

Expansion of oil-fired (OP) and gas-fired (GP) plants are not included as decision variables, as they just account for small proportions and China lacks oil and gas resources. Hydro and nuclear are not included as well, since their long-term expansion plans have almost been set by the government. it is not realistic to incorporate too many changes on them.

2) Trading Mechanisms Involved: $\mathrm{CDM}$ and emission trading (ET) are included as major carbon trading mechanisms. Assuming that China would commit to reduce $\mathrm{CO}_{2}$ emission in 2020 , then CDM is the only active mechanism before 2020 , and

$$
\begin{cases}\sum_{y \in Y} \sum_{m \in U}\left(X_{m, y}^{G} \cdot e_{m, y}^{G}\right)-\sum_{n \in T} \sum_{i=1}^{\max \left(Y_{n}^{P}, y-1\right)} X_{n, y-i}^{E} \leq E^{T} & \\ \sum_{m \in U}\left(X_{m, y}^{G} \cdot e_{m, y}^{G}\right)-\sum_{n \in T} \sum_{i=1}^{\max \left(Y_{n}^{P}, y-1\right)} X_{n, y-i}^{E} \leq D_{y} \cdot e_{y}^{T} \quad y \in Y_{E} \\ \sum_{m \in U}\left(X_{m, y}^{G} \cdot e_{m, y}^{G}\right)-\sum_{n \in T} \sum_{i=1}^{\max \left(Y_{n}^{P}, y-1\right)} X_{n, y-i}^{E} \leq E_{0}^{T}\left(1-r_{y}^{E}\right) & y \in Y_{E}\end{cases}
$$


TABLE II

TeChNOLOgy Cost AND Performance Data

\begin{tabular}{|c|c|c|c|c|c|c|c|c|c|c|c|c|c|c|c|}
\hline \multirow{2}{*}{ Technology } & \multicolumn{3}{|c|}{ Efficiency (in \%) } & \multicolumn{3}{|c|}{ Capital $\operatorname{cost}(\$ / \mathbf{k w})$} & \multicolumn{3}{|c|}{ Fixed $O \& M \operatorname{cost}(\$ / \mathbf{k w})$} & \multicolumn{3}{|c|}{ Production $\operatorname{cost}(\$ / \mathrm{MWh})$} & \multicolumn{3}{|c|}{$\mathrm{CO}_{2}$ intensity $(\mathrm{kg} / \mathrm{MWh})$} \\
\hline & 2010 & 2020 & 2030 & 2010 & 2020 & 2030 & 2010 & 2020 & 2030 & 2010 & 2020 & 2030 & 2010 & 2020 & 2030 \\
\hline$S C$ & 43.0 & 45.0 & 46.5 & 522 & 497 & 472 & 35 & 33 & 31 & 21 & 20 & 19 & 75.0 & 72.0 & 69.0 \\
\hline$S C \& C C R$ & 43.0 & 45.0 & 46.5 & 538 & 505 & 480 & 35 & 33 & 31 & 21 & 20 & 19 & 75.0 & 75.0 & 69.0 \\
\hline$S C \& C C S$ & 35.0 & 39.3 & 41.4 & 848 & 692 & 632 & 58 & 47 & 43 & 26 & 23 & 22 & 9.0 & 8.5 & 8.0 \\
\hline SC Ret & 31.6 & 37.2 & 39.6 & 403 & 231 & 174 & 60 & 48 & 43 & 29 & 25 & 23 & 10.0 & 9.0 & 8.4 \\
\hline$S C \& C C R$ Ret & 33.5 & 38.3 & 40.6 & 374 & 209 & 160 & 57 & 46 & 43 & 27 & 24 & 22 & 9.4 & 8.7 & 8.2 \\
\hline$U C$ & 45.8 & 48.0 & 49.5 & 537 & 485 & 462 & 36 & 32 & 31 & 20 & 18 & 17 & 70.0 & 67.0 & 65.0 \\
\hline$U C \& C C R$ & 45.8 & 48.0 & 49.5 & 552 & 494 & 469 & 36 & 32 & 31 & 20 & 18 & 17 & 70.0 & 67.0 & 65.0 \\
\hline$U C \& C C S$ & 37.3 & 41.6 & 43.9 & 872 & 686 & 626 & 59 & 46 & 42 & 25 & 21 & 19 & 8.6 & 8.0 & 7.5 \\
\hline UC Ret & 33.7 & 39.1 & 42.3 & 403 & 231 & 174 & 62 & 47 & 43 & 27 & 22 & 20 & 9.5 & 8.5 & 7.8 \\
\hline$U C \& C C R$ Ret & 35.6 & 40.4 & 43.2 & 374 & 209 & 160 & 59 & 46 & 42 & 26 & 21 & 20 & 9.0 & 8.2 & 7.6 \\
\hline$I G C C$ & 40.0 & 46.0 & 52.0 & 1118 & 705 & 606 & 41 & 26 & 23 & 23 & 17 & 15 & 81.0 & 69.0 & 59.0 \\
\hline$I G C C \& C C R$ & 40.0 & 46.0 & 52.0 & 1176 & 737 & 631 & 41 & 26 & 23 & 23 & 17 & 15 & 81.0 & 69.0 & 59.0 \\
\hline$I G C C \& C C S$ & 36.0 & 41.5 & 46.2 & 1400 & 874 & 744 & 46 & 29 & 25 & 25 & 18 & 16 & 8.9 & 8.1 & 7.3 \\
\hline IGCC Ret & 32.5 & 39.5 & 45.8 & 348 & 215 & 168 & 49 & 30 & 26 & 28 & 20 & 17 & 9.9 & 8.7 & 7.7 \\
\hline$I G C C \& C C R$ Ret & 33.5 & 39.9 & 45.4 & 299 & 193 & 146 & 46 & 29 & 25 & 27 & 19 & 17 & 9.6 & 8.4 & 7.4 \\
\hline Wind & -- & -- & -- & 1251 & 923 & 834 & 37 & 27 & 25 & 0 & 0 & 0 & 0.0 & 0.0 & 0.0 \\
\hline
\end{tabular}

ET would be introduced after 2020. In CDM, qualified plants would trade their allowances by means of long-term contracts, with ten years as fixed validity period. $p_{m, y}^{\max }$ for CDM is set $20 \%$ in 2011, then increased to $100 \%$ in 2020 gradually. Generally, emission reduction obligations would be introduced with ET; 2010 is set as the benchmark year; reduction obligation is set $0 \%$ in 2020, and $10 \%$ in 2030. $p_{m, y}^{\max }$ for ET is set $100 \%$ since its first introduction in 2020, thus the terms of allowance penalty could be ignored. Carbon price is set $10 \$ /$ ton for CDM and $15 \$ /$ ton for ET. Growth rate for the prices are 3\%, the same as deflating rate. All the cost/price data in this paper have been converted to present values by the deflating rate.

\section{B. Basic Data}

Power demand in 2010 is $3430 \mathrm{TWh}$, with annual growth rate as $3.1 \%$ on average [15]. Installed capacity in 2010 is $920 \mathrm{GW}$ with $681 \mathrm{GW}$ fired-based, $194 \mathrm{GW}$ hydro-based, $30 \mathrm{GW}$ wind and $13 \mathrm{GW}$ nuclear. Considering retirement schedule of existing plants and the government's agenda of "closing down small thermal power plants", $288 \mathrm{GW}$ of fired-based capacity will be out of service until 2030, taking up about $42.3 \%$.

Data on most of the candidate plant sets are presented in Table II [15]-[20]. It should be noted that some of the data are referenced by investigations with engineers in industry; and some are obtained in informal reports of demonstration projects. The cost data referenced by international experiences have been adjusted according to price differences between countries. Cost for $\mathrm{CO}_{2}$ transportation and storage is set as $5.9 \$$ ton. Upper and lower bounds of YGH for SC, UC and IGCC are set 8000 and $2000 \mathrm{~h}$; while for conventional coal-fired plants, the upper bounds would decline from 6000 to $2000 \mathrm{~h}$ gradually, embodying the government's policies of "low-efficiency power plants replacement". Cap on primary coal for generation is set $1.3 \mathrm{Gt}$ in 2010, with an average annual growth rate of $3 \%$, and the cap of import proportion is $10 \%$. Maximum

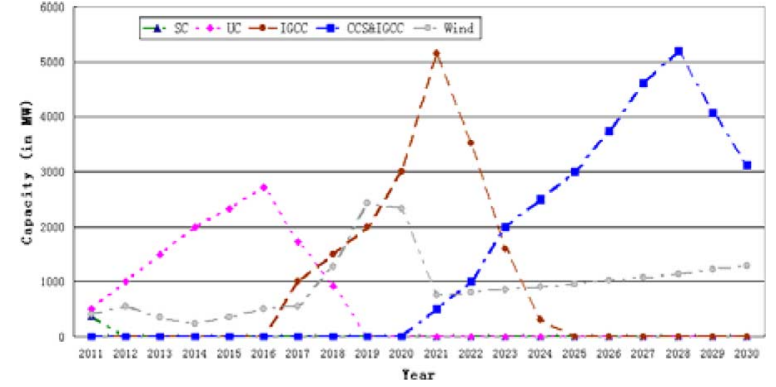

Fig. 2. Capacity expansion plan from 2011 to 2030.

exploitable capacities for wind are 30,120 and $220 \mathrm{GW}$ in 2010, 2020 and 2030. Besides, capacity expansion plans of other sets of plants are decided according to China's 11th and 12th Five-Year Plan as well as Long-Term Developing Plan of Economy, which could be seen in Table I. IGCC and CCS would be commercialized after 2015 and 2020, with five-year transition periods [21]. The maximum fluctuation rate for expanded capacities between years is set as $20 \%$.

In the case study, the basic settings above would be referred as "normal scenario".

\section{Results and Discussions}

1) Generation Capacity Expansion Plan: Fig. 2 shows the plan of PGE from 2011 to 2030. Wind is always competitive during the planning period due to its advantages in low cost and cleanness. Before 2016, UC is the first choice for expansion; however, from $2017 \sim 2020$, regular IGCC takes the lead and the expansion of UC is going down; then declining trend could be observed on regular IGCC after 2020, when CCS begins to be available and largely developed.

The evolution of power mix is shown in Fig. 3. Remarkable reduction could be observed on conventional coal-fired plants, from $43 \%$ to $17 \%$; SC shows a declining trend as well, though 


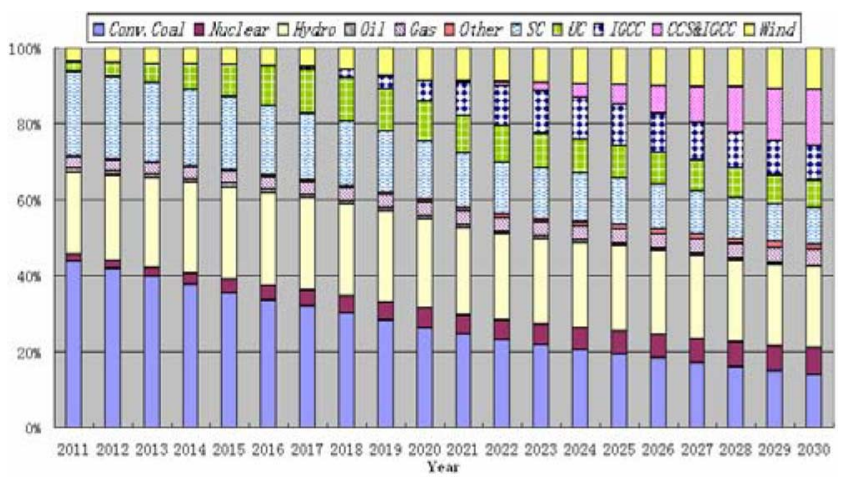

Fig. 3. Capacity proportion for different types of plants.

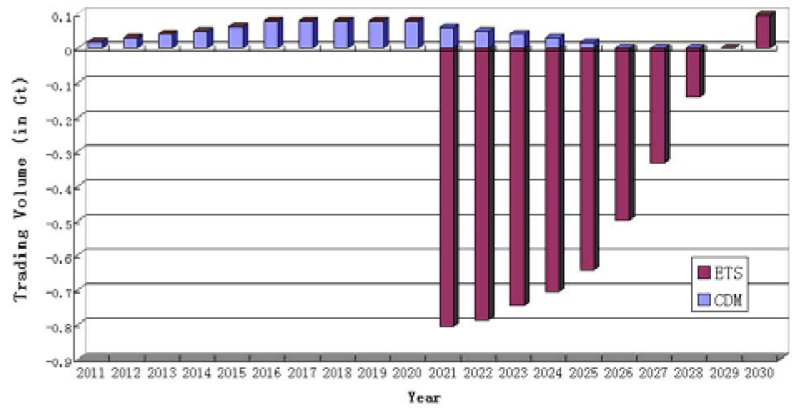

Fig. 4. $\mathrm{CO}_{2}$ trading strategies.

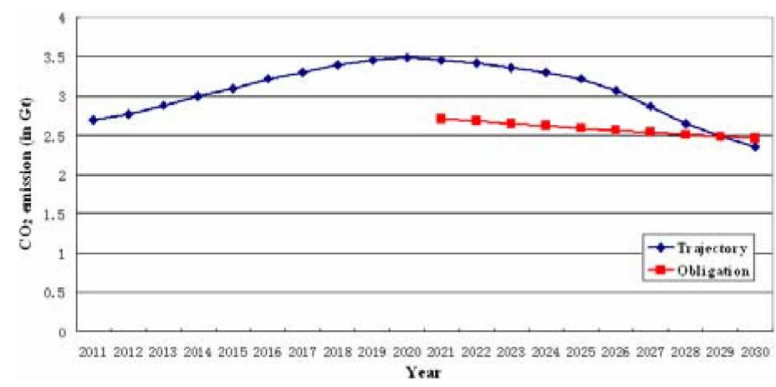

Fig. 5. $\mathrm{CO}_{2}$ emission trajectory.

much slower. The proportions of wind and UC grow rapidly in the first few years, then wind keeps steady and UC begins to decrease after 2016. Afterward, IGCC begins to show its critical role in PGE; the proportion grows rapidly from $0 \%$ to $23 \%$, including $13.6 \%$ with CCS and $9.4 \%$ without CCS.

2) $\mathrm{CO}_{2}$ Trading Strategies: Fig. 4 shows the overall trading allowances in CDM and ET. Volumes in CDM increase until 2017, and start decreasing after 2020, when ET is ready. In fact, no more new CDM contracts are signed after 2020 with the occurrences of allowance shortfall; the remaining sold volumes in CDM after 2020 are from the execution of the signed contracts before 2020. The allowance shortfall is huge under the set reduction obligations, leading to great economic losses to China's power sector. Total allowance sold in CDM is $8.0 \mathrm{Gt}$, while that bought in ET is $45.6 \mathrm{Gt}$. The results indicate serious prospects of $\mathrm{CO}_{2}$ reduction that confronted by China.

3) $\mathrm{CO}_{2}$ Emission Trajectory: Curves in Fig. 5 depict $\mathrm{CO}_{2}$ emission trajectory regardless of traded allowances, and the set reduction obligations. Peak emission would hit the height of

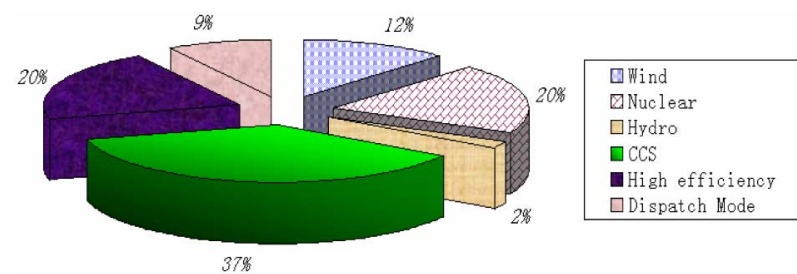

Fig. 6. Contributors of $\mathrm{CO}_{2}$ emission reduction.

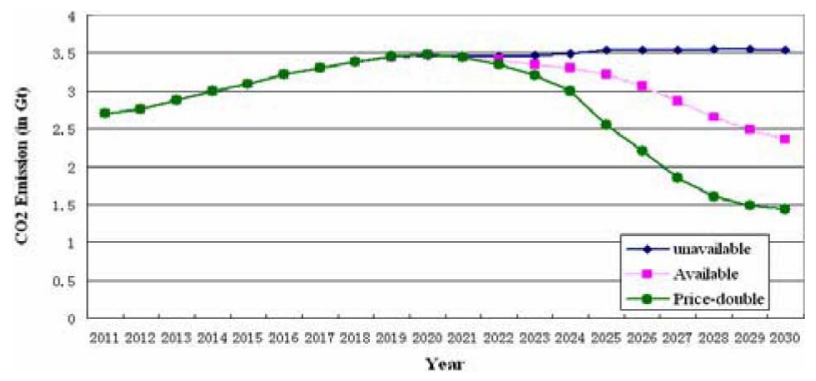

Fig. 7. $\mathrm{CO}_{2}$ trajectories in different scenarios for CCS.

$3.48 \mathrm{Gt}$ in 2020 , far beyond the expected obligation; and finally drop to $2.36 \mathrm{Gt}$ in 2030 , which is $14.5 \%$ below the base level in 2010. However, the obligations could finally be met after 2029 .

4) Contributors of $\mathrm{CO}_{2}$ Emission Reduction: Emission intensity in 2010 is $0.64 \mathrm{~kg} / \mathrm{kwh}$. If the same intensity is kept, the emission in 2030 would be $5.21 \mathrm{Gt}$. However, by adopting various mitigation actions, $2.85 \mathrm{Gt}$ has been cut, which accounts for about $55 \%$. The reduction is mainly due to the contributors listed in Fig. 6. CCS contributes about 1/3; the changing in power mix, including nuclear, wind and hydro, contributes almost another $1 / 3$. Improvements in generation efficiency take up $20 \%$, while the remaining $9 \%$ is from the changing of dispatching mode (by adopting the scheme in which plants with lower production costs would be scheduled on in priority, instead of the traditional scheme based on average dispatching).

5) Penetration of CCS: CCS is the most promising option for $\mathrm{CO}_{2}$ abatement. Two extreme conditions are studied to reveal the potential of CCS and its impacts on emission trajectory, which is shown in Fig. 7. Firstly, If CCS would be unavailable during the whole planning period; no evidence of reduction could be expected, with total emission as $66.4 \mathrm{Gt}$ and 3.54 Gt in 2030, increased by $7.5 \%$ and $33.3 \%$ compared with the normal scenario. Then, if carbon price is doubled, $497 \mathrm{GW}$ of carbon capture plants would be installed, taking up $23.3 \%$ in power mix; however, the numbers are $219 \mathrm{GW}$ and $10.9 \%$ in the normal scenario. $\mathrm{CO}_{2}$ emission is significantly cut as well, which would be as low as $55.4 \mathrm{Gt}$ totally and $1.43 \mathrm{Gt}$ in 2030 , decreased by $10 \%$ and $40 \%$. The analysis above provides a rough estimation on upper and lower bounds of CCS penetration and corresponding emission trajectories.

6) Discussions on $\mathrm{CO}_{2}$ Reduction Obligation: Reduction obligation is a "soft" scheme, with carbon price as penalty for excessive emission. With the set obligations, total expense in $\mathrm{CDM}$ and ET is as much as $67.1 \mathrm{G} \$$. However, the losses could be significantly cut or even turned over with the delaying of the first enforcing year of reduction obligations, which could be seen in Fig. 8. 


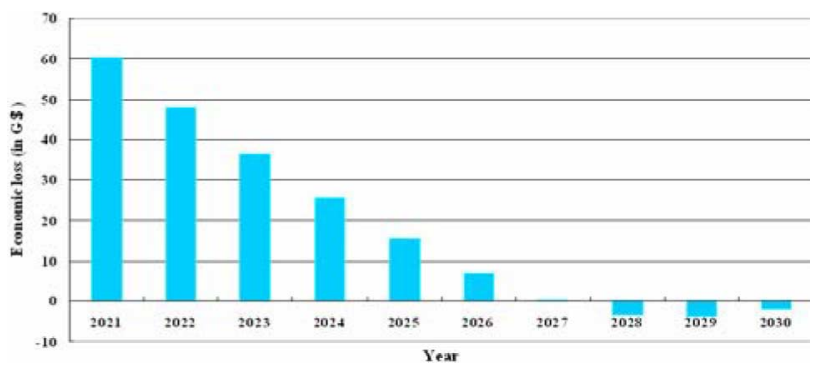

Fig. 8. $\mathrm{CO}_{2}$ trading expenses with different first enforcing year.

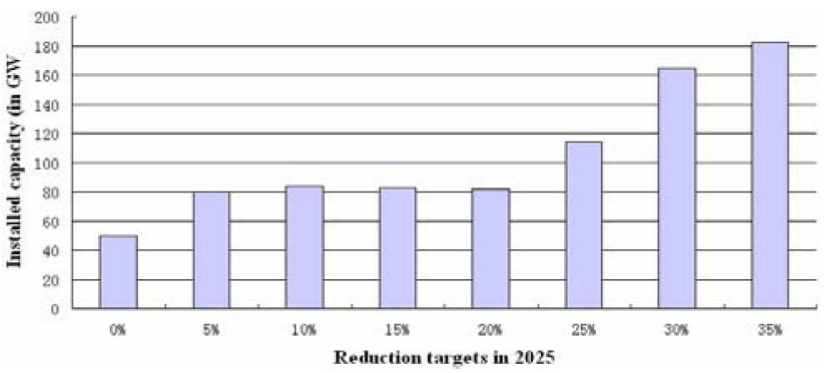

Fig. 9. Capacity of CCR and CCS retrofit under different reduction targets.

7) Discussions on CCR and Plant Retrofit: CCR and CCS retrofit are seldom utilized in the normal scenario. However, if the imposed year of reduction targets is advanced (for example, 2025 instead of 2030, as seen in Fig. 9), the direct utilization of CCS would not be "timely" enough. Hence, plenty of in-service plants should be treated as CCR first; then after CCS technologies become technical and commercial mature, large-scale of CCS retrofit will be enforced. The results shown in Fig. 9 imply that quick actions like CCR should be taken if quick reduction is required.

\section{CONCLUSION}

An integrated PGE planning model toward LCPGE is proposed in this paper, which appropriately incorporates and formulates the impacts of various low-carbon factors. A compromised modeling approach is presented, adapting to the characteristics of the LCPGE model. With the approach, general mixed integer nonlinear PGE planning problems could be formulated as standard linear models, which remarkably decreases the complexities while maintains proper precision degree of modeling. The LCPGE model is implemented to gain insights into the optimal PGE plan for China's power sector. Trajectory of $\mathrm{CO}_{2}$ emission is depicted, main contributors in emission reduction are identified, and economic tradeoffs in $\mathrm{CO}_{2}$ abatement are evaluated.

The research in this paper is a new explore especially in the formulation of low-carbon factors in PGE model. Lots of more efforts should be made to improve the model and the modeling approach, for example, including production simulations or incorporating stochastic issues. These works would be carried out in the following research. Hopefully, the paper could provide new ideas in the research area of developing a low-carbon power sector.

\section{REFERENCES}

[1] IPCC, "Intergovernmental panel for climate change fourth assessment report," . Cambridge, U.K., Cambridge Univ. Press, 2007.

[2] N. Stern, The Economics of Climate Change: The Stern Review. Cambridge, U.K.: Cambridge Univ. Press, 2007.

[3] Committee on Climate Change, "Building a low-carbon economyThe UK's contribution to tackling climate change," . London, U.K., Dec. 2008, The Stationary Office.

[4] Secretary of State for Trade and Industry, "Our energy future-creating a low carbon economy,” . London, U.K., Feb. 2003, Energy White Paper.

[5] M. Grubb, T. Jamasb, and M. G. Pollitt, Delivering a Low-carbon Electricity System. Cambridge, U.K.: Cambridge Univ. Press, 2008.

[6] J. Sirikum, A. Techanitisawad, and V. Kachitvichyanukul, "A new efficient GA-benders' decomposition method: For power generation expansion planning with emission controls," IEEE Trans. Power Syst., vol. 22, no. 3, pp. 1092-1100, Aug. 2007.

[7] J. L. C. Meza and M. B. Yildirim, "A model for the multi-period multiobjective power generation expansion problem," IEEE Trans. Power Syst., vol. 22, no. 2, pp. 871-878, May 2007.

[8] S. C. Srivastava, A. K. Srivastava, and U. K. Rout, "Least cost generation expansion planning for a regional electricity board in India considering green house gas mitigation," presented at the Power Con 2000 on Power System Technology, Dec. 4-7, 2000, vol. 1, pp. 31-36.

[9] R. Doherty, H. Outhred, and M. O'Malley, "Generation portfolio analysis for a carbon constrained and uncertain future," in Proc. Int. Conf. Future Power Systems, Amsterdam, The Netherlands, Nov. $11-18,2005$.

[10] Z. Yu, "Planning wind power considering system reliability in peak demand time," presented at the IEEE PES General Meeting on Conversion and Delivery of Electrical Energy in the 21st Century, Jul. 20-24, 2008.

[11] H. Wang and T. Nakata, "Analysis of the market penetration of clean coal technologies and its impacts in China's electricity sector," Energy Pol., vol. 37, pp. 338-351, 2009.

[12] M. Van Den Broek, A. Faaij, and W. Turkenburg, "Planning for an electricity sector with carbon capture and storage-case of the Netherlands," Int. J. Greenhouse Gas Control, vol. 2, pp. 105-129, 2008.

[13] M. A. Uyterlinde, M. Junginger, and H. J. De Vries, "Implications of technological learning on the prospects for renewable energy technologies in Europe," Energy Pol., vol. 35, pp. 4072-4087, 2007.

[14] K. Riahi, E. S. Rubin, and L. Schrattenholzer, "Prospects for carbon capture and sequestration technologies assuming their technological learning," Energy, vol. 29, pp. 1309-1318, 2004.

[15] IEA, "World energy outlook," 2007. Paris, France, 2007.

[16] IEA, "Prospects for capture and storage," 2004. [Online]. Available: http://www.iea.org/Textbase/Publications/free_new_Desc.asp?PUBS _ID=1466.

[17] M. C. Bohn, H. J. Herzog, and J. E. Parsons, "Capture-ready coal plants-options, technologies and economics," Int. J. Greenhouse Gas Control, vol. 1, pp. 113-120, 2007.

[18] P. Viebahn, J. Nitsch, and M. Fischedick, "Comparison of carbon capture and storage with renewable energy technologies regarding structural, economic, and ecological aspects in Germany," Int. J. Greenhouse Gas Control, vol. 1, pp. 121-133, 2007.

[19] X. Liang, D. Reinera, and J. Gibbins, "Assessing the value of $\mathrm{CO}_{2}$ capture ready in new-build coal-fired power plants in China," presented at the Int. Conf. GHGT-9, 2008.

[20] T. Jamasb, W. J. Nuttall, and M. G. Pollitt, Future Electricity Technologies and Systems. Cambridge, U.K.: Cambridge Univ. Press, 2008.

[21] J. Gibbins and H. Chalmers, "Preparing for global rollout: A 'developed country first' demonstration programme for rapid CCS deployment," Energy Pol., vol. 36, pp. 501-507, 2008.

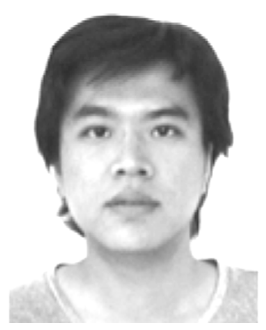

Qixin Chen received the B.S. degree from the Department of Electrical Engineering in Tsinghua University, Beijing, China, 2005, where he is currently pursuing the $\mathrm{Ph} . \mathrm{D}$. degree.

His research interests include low-carbon electricity, power system economics and optimization, power market, and power generation expansion planning. 
Chongqing Kang (M'01-SM'07) received the Ph.D. degree from the Department of Electrical Engineering in Tsinghua University, Beijing, China, in 1997.

$\mathrm{He}$ is now a Professor at the same university. His research interests include low-carbon electricity, power system planning, power market, power system reliability, and power system load forecasting.

Qing Xia (M'01-SM'08) received the Ph.D. degree from the Department of Electrical Engineering in Tsinghua University, Beijing, China, in 1989.

He is now a Professor at the same university. His research interests are mainly in power economics, power market, power system expansion planning, power system reliability, power system load forecasting, and smart grid.
Jin Zhong (S'00-M'05) received the Ph.D. degree from Chalmers University of Technology, Gothenburg, Sweden, in 2003.

At present, she is an Assistant Professor in the Department of Electrical and Electronic Engineering, The University of Hong Kong. Her areas of interest are electricity sector deregulation, ancillary service pricing, power system planning, and smart grid. 